\title{
STRATEGIES FOR CONTROL, PREVENTION AND ELIMINATION OF URBAN RABIES IN WEST AFRICA
}

\author{
AIYEDUN, J.O. ${ }^{1}$, OLUDAIRO, O.O. ${ }^{1}$, OLORUNSOLA, I.D. ${ }^{2}$ and OKOLI, C.P. ${ }^{3}$ \\ ${ }^{1}$ Department of Veterinary Public Health and Preventive Medicine, Faculty of Veterinary Medicine, University of Ilorin \\ ${ }^{2}$ Department of Veterinary Microbiology, Faculty of Veterinary Medicine University of Ilorin \\ ${ }^{3}$ Department of Veterinary Medicine, Faculty of Veterinary Medicine University of Abuja
}

Received: 12 February 2017; $\quad$ Accepted: 19 April 2017

\begin{abstract}
Rabies is a viral disease of global threats, almost $100 \%$ fatalities in any incidence, killing 15,200 to 159,000 human beings annually. About $70 \%$ of the world's population resides in areas where dogs are reservoirs for rabies, particularly in the developing countries where the incidence of human and canine rabies is unknown. The goal of this review is to create awareness on the current burden and strategies on control, prevention and elimination of rabies in West Africa. There is need for a strong political and technical commitment to control rabies in West Africa. In lieu of the re-emergence, further research on the current vaccine and other possible control and elimination strategies are recommended.
\end{abstract}

Key words: Strategies, Urban rabies, Control, Prevention, Elimination, West Africa.

\section{INTRODUCTION}

Rabies is the oldest and commonest communicable disease of humans (Wilkinson, 1988). Rabies is present in all continents and is endemic in most African and Asian countries (Ogunkoya et al, 1984; Aiyedun, 2011). It is an acute viral encephalomyelitis that affects all warm-blooded animals and humans, and is almost always fatal once symptoms occur (WHO, 2010). It is caused by a neurotropic RNA virus, which belongs to the genus Lyssavirus of the family Rhabdoviridae, and is found mostly in the brain, spinal cord, salivary glands, and saliva of the affected animals (McElhinney et al, 2008).

Rabies is transmitted usually through bite and virusrich saliva of an infected animal. Transmission by non-salivary routes has been recorded (Milius et al, 2004). These include aerosol transmission to humans in the laboratory, in bat-infested caves and organ transplant. In most cases, humans contract the disease from stray domestic animals. However, animals contract the disease through wounds, when they are bitten by another animal infected with rabies. The

Corresponding author: Dr. OLUDAIRO, O.O.

E-mail address: olaaiyedun@yahoo.com; oludairo@ hotmail.com Present address: Department of Veterinary Public Health and Preventive Medicine, Faculty of Veterinary Medicine University of Ilorin most dangerous wounds are those close to the head or wounds that do not bleed. The incubation period is prolonged and highly variable, depending on the quantity of the inoculum and the site of the bite, being shorter after a bite near the brain than following a more distant one (Zienius et al., 2003; Milius et al, 2004). Rabies has been associated with animal bites for more than 3000 years. Domestic dogs (canis lupus familiaris) are the most important vectors of human rabies worldwide (Knobel et al, 2005; Aiyedun, 2011).

African and Asian countries are particularly affected by rabies because of the abundance of animal reservoirs, inadequate healthcare and control measures (Fooks, 2004; Knobel et al., 2005). In Africa and Asia, human mortality from endemic canine rabies was estimated to be $15,200-159,000$ annually. It is the eleventh $\left(11^{\text {th }}\right)$ killer disease of the world (WHO, 2010). About fourteen million people receive costly post-exposure prophylaxis each year globally after having been bitten by rabid or suspected rabid animals (WHO, 2010). In these parts of the world, modern rabies vaccines and immunoglobulin are usually in short supply and their high costs often prevent their use by those most in need of it (Aiyedun and Olugasa, 2013).

The greatest challenge to rabies control worldwide is the extent of the dog rabies epizootics in Asia and Africa, about $98 \%$ of human rabies occurs in this region with large number of stray and domestic dogs 
(Warrel and Warrel, 2004). High prevalence of human rabies has always correlated well with stray dog population (Aiyedun and Olugasa, 2012). Efforts to eliminate such stray dogs in order to control dogmediated rabies have often been unsuccessful. This is because the so-called stray dogs are often owned by an individual or group who fail to control them. They are perceived to be useful for their garbage removal, sanitary control and their guard role. Therefore, there is frequent resistance to their elimination (Wilsmore et al, 2006).

\section{Strategies for Rabies Control and prevention in West Africa}

Rabies is one of the zoonoses that continue to pose global public health challenge in terms of its control and eradication, although it is readily preventable with vaccines if applied appropriately at the right time. Prevention of rabies is relatively straightforward and consists of a primary strategy aimed at avoiding interactions with possibly rabid animals and, for some persons at greatest and unavoidable risk, primary vaccination and a secondary defense after an exposure has occurred (Ratzan, 2006).

Generally, veterinary vaccines are used for preexposure treatment, while human vaccines, which are considerably more expensive to produce, are used for post-exposure. Pre-exposure prophylaxis may be recommended in people at high risk (Bishop et al, 2003). Treatment of clinically infected animals and man is of no avail, post exposure treatment of man with vaccine and hyperimmune serum is used successfully (Wilsmore et al, 2006). Vaccines are used successfully against rabies and can give up to three years immunity, although vaccination does not preclude the possibility of rabies infection, as confirmed cases of rabies have occurred in previously vaccinated dogs and cats (Okonko et al., 2010). Nevertheless, they provide a high level of protection. The protection they can offer is measured by the antibody level they stimulate. The WHO standard is that a neutralizing antibody level of $0.5 \mathrm{eu} / \mathrm{ml}$ and above (measured against an international standard serum) is considered threshold immunity, although antibody responses have not been fully equated with protection (Wilsmore et al, 2006).

Pre-exposure vaccination constitutes the main solution that can be used to prevent canine rabies and interrupt rabies transmission cycle (WHO, 2010). Effective rabies control in any country demands 80$85 \%$ of the dog's population immunized (Beran, 1982). The World Health Organization recommends $80 \%$ minimum vaccination coverage of the population at risk in all areas (WHO, 2010).

Low and high egg passage (LEP, HEP) Flury rabies vaccines for dog and cat respectively, have been produced in Nigeria by the National Veterinary
Research Institute (NVRI) since 1956 and are the common prophylaxes for animal rabies control in Nigeria and some other West Africa countries. Initial vaccination of dogs is at 3 months of age. Immunity lasts for 3 years but an annual booster vaccination is recommended in Nigeria because of the enzootic nature of rabies in most areas (Adeyemi and Zessin, 2000). Vaccination in already infected animals does not significantly alter the clinical picture or development time of the disease (Fooks et al, 2004). This is in contrast to prompt post-exposure prophylaxis in man, which is effective after natural infection. It is possible that an animal infected before vaccination would continue to incubate the disease despite developing a high antibody titer (Fooks et al, 2004; Olugasa et al, 2011).

\section{Problems associated with rabies vaccination}

Rabies vaccines have become costly and scarce in recent times. Also, standardized quality control of LEP rabies vaccine for dog produced in Nigeria is lacking (Adeyemi and Zessin, 2000). Human rabies vaccines in use today are based on inactivated live virus, and are thought to work by eliciting neutralizing antibodies to envelope glycoprotein. These vaccines are expensive to prepare and carry the theoretical risk of transmission of live virus if inadequately inactivated (CDC, 2008).

With rabies vaccines, injection site reactions, such as pain, erythema, and swelling or itching at the site, are the most commonly reported adverse events. According to the recommendations of the CDC's Advisory Committee on Immunization Practices (ACIP), once begun, rabies vaccination should not be interrupted or halted due to local or mild systemic adverse reactions.

Anti-inflammatory and antipyretic agents such as ibuprofen or acetaminophen can help manage these reactions. Rabies vaccines should be given with caution in old aged patient (CDC, 2008).

\section{Quarantine}

If the animal causing the potential exposure is a dog, cat or ferret and is available, it will generally be kenneled for a period of 10 days and observed. If the animal is alive and healthy after that time, the animal is considered not to have been shedding rabies virus in its saliva at the time of the encounter (Tepsumethanon et al, 2004).

\section{Stray dog control}

Control of stray dogs, wildlife reservoirs and mass education of the public are necessary approaches for rabies control measures to be effective (Aiyedun, 2011). Efforts to eliminate stray dogs in order to control dog-mediated rabies have often been unsuccessful, particularly in developing countries (Wilsmore et al., 2006; Aiyedun and Olugasa, 2012). 
This is because the so-called stray or feral dogs are often owned by an individual or group but not controlled. Thus, there is frequent resistance to their elimination. Stray/feral dog population control may be achieved to some extent by neutering owned dogs because life span of stray/feral dogs is short with fewer chances of breeding compared with owned dogs which, if not neutered, breed excessively to increase the stray dog population (Wilsmore et al., 2006).

\section{New Developments in Rabies Control}

Modern research aims to reach free-roaming dogs by oral vaccination, thus limiting problems associated with dog elimination (Mantovani and Marabelli, 2004). The development of oral vaccines has enabled the eradication of rabies from the red fox population throughout most of Europe. In addition, programmes for oral vaccination of wildlife are being undertaken in North America (Wilsmore et al, 2006). If the results of rabies control in dogs by oral vaccination can match those achieved in the red fox population, it should help in eliminating rabies from where it is most dreadful: the urban habitat of developing countries (Pastoret et al., 2004). In the Philippines, the results of a dog oral vaccination campaign, using local-made boiled intestine bait containing a capsule filled with SAD B19 vaccine demonstrated that oral vaccination of dogs against rabies is a promising supplementary method for dog rabies control (Wilsmore et al., 2006).

Oral rabies vaccination programmes have been implemented to control the spread of wildlife rabies, yet the current surveillance systems are inadequate for the efficient management and evaluation of these large scale vaccine baiting programmes (Blanton et al., 2007). Progress has been made in preventing the spread of raccoon rabies from the Eastern United States. Nevertheless, the most profound challenge facing the programme is the need for baits and oral vaccine with improved effectiveness in other mesocarnivore reservoir species, such as skunks and monogoose (Velasco-Villa et al., 2008). Oral vaccine can be safely distributed in baits in West Africa, but expensive with many logistics problems such as climatic factors, topography and distribution network (Madhusudana et al., 2002).

Initiation of rabies vaccination after onset of clinical symptoms in patients with confirmed rabies diagnoses is not recommended and might be detrimental. Survival has been well documented for only few patients especially those that had received rabies vaccination before the onset of disease (Ajayi et al., 2006).

As a routine, more than one assay system should be employed simultaneously to increase the chances of making a rabies diagnosis and increase confidence in negative results. There are no clinical or gross pathognomonic lesions for the diagnosis of rabies in animals; therefore, confirmation of infection can only be achieved by laboratory techniques (OIE, 2004). Diagnostic techniques for rabies in animals have been internationally standardized, and the hippocampus, cerebellum and the medulla oblongata are the recommended brain tissue of choice (OIE, 2004).

\section{Conclusion and Recommendation}

Rabies is almost uniformly fatal, it is readily preventable with currently available tools. However, asses to these tools and knowledge of their proper use are often limited, especially in developing countries. Nevertheless, successful strategies for canine rabies elimination are been pursued, even in such areas. These strategies are diagnostic and laboratory based surveillance systems, effective canine population management, education and awareness, legislation and government support as well as intersectorial and multisectorial collaborations. These should be implemented in a coordinated and concurrent manner. There is need for a strong political and technical commitment to control rabies in West Africa. These plans should have specific goals, measurable outcomes and conducted under the guidance of supportive government in collaboration with international partners and non-governmental organizations.

Government should be encouraged to establish citywide rabies elimination committees. Public health sector should take leading role in the activities of the committee, with close involvement of nongovernmental organisations and private sector. Efforts should be made to fully incorporate rabies control activities at all levels of the healthcare services, integrating them with other public health programmes such as expanded programme on immunization and those for tuberculosis and vector borne diseases. In this manner, it is possible to synergize programmes for efficient use of human, material and financial resources.

An organized delivery of mass antirabies vaccination campaigns, using Geographic Information System focusing techniques to achieve effective and efficient delivery, regular monitoring and strict enforcement of rabies control regulations, dog movements and population control in West Africa be adopted. The vaccination coverage needs to be improved upon by subsidizing or making antirabies vaccination free to increase compliance and invariably reduce human rabies death rate in the West Africa sub region. The public should be given basic education on first aid care and prophylaxis treatments of bite victims. Human rabies vaccine should be made readily available and less expensive. There is the need for seroprofile evaluation at the community level periodically to know the status of dogs at that level. 


\section{REFERENCES}

Adeyemi, I. and Zessin, K. (2000): Retrospective dog rabies vaccination evaluation at the University of Ibadan, Nigeria (1988-1992) Veterinary Arhiv 70 (5); 223-230.

Aiyedun, J.O. (2011): Epidemiology of Canine Rabies in Ilorin, Nigeria. A PhD Thesis in the Department of Veterinary Public Health and Preventive Medicine, University of Ibadan, Ibadan Nigeria pp248.

Aiyedun, J.O. and Olugasa, B.O. (2012): Use of Aerial Photograph to enhance Dog Population Census in Ilorin, Nigeria. Sokoto Journal of Veterinary Sciences, 10 (1), 28-32.

Aiyedun, J.O. and Olugasa, B.O. (2013): Level of Compliance with Vaccination against Rabies among Dogs in Ilorin, Nigeria. International Journal of Applied Research and Technology. 2 (6) $158-162$

Ajayi, B.B.; Rabo, J.S. and Baba, S.S. (2006): Apparently healthy dogs: histological and immunological studies. The Nigerian post graduate Medical Journal. 13 (2); 125-134.

Beran, G.W. (1982): Ecology of dog in central Philippines in relation to rabies control effort. Comparative Immunology, Microbiology and Infectious Disease. 5; 265-270.

Bishop, G.C.; Durrheim, D.N.; Kloeck, P.E.; Godlonton, J.D.; Bingham, J.; Speare, R. and Rabies Advisory group (2003): Rabies: Guide for the Medical, Veterinary, and Allied Professions. $2^{\text {nd }}$ print. ISBN 1-86871-119-6. Department of Agriculture and Department of Health, South Africa.

Blanton, J.D.; Hanlon, C.A. and Rupprecht, C.E. (2007): Rabies surveillance in the United States during 2006. Journal of the American Veterinary Medical Association 231; 540-56.

Center for Disease Control and Prevention (2008): Human Rabies Prevention - United States, 2008. Recommendations of the Advisory Committee on Immunization Practices. Morbidity Mortality Weekly Report. 57; 205210.

Fooks, A.R.; McElhnney, L.M.; Mariston, D.A.; Selden, D.; Jollff, T.A.; Wackeley, P.R.; Johnson, N.I. and Brookes, S.M. (2004): Identification of a European bat lyssavirus type 2 in a Daubenton's bat (Myotis daubentoni) found in staines. Surrey United Kingdom. Veterinary Record. 155; 430-455.

Fooks, A.R. (2004): The challenge of Emerging Lyssaviruses. Expert Review. Vaccines. 3; 8992.

Knobel, D.L.; Cleaveland, S.; Coleman, P.G.; Fevre, E.M.; Meltzer, M.I.; Miranda, M.E.G.; Shaw, A.; Zinsstag, J. and Meslin, F.X. (2005): Reevaluating the burden of rabies in Africa and Asia. Bulletin of World Health Organization.
$83(5) ; 360-368$

Madhusudana, S.N.; Nagaraj, D.; Uday, M.; Ratnavalli, E. and Kumar, M.V. (2002): Partial recovery from rabies in a six-year-old girl. International Journal of Infectious Diseases 6; 85-86.

Mantovani, A. and Marabelli, R. (2004): Dog rabies past and present, in the Mediterranean Basin. In: Historical Perspective of rabies in Europe and the Mediterranean basin. (Eds.), A.A. King, A.R. Fooks, M. Aubert and A.I. Wandeler. Paris, OIE: 215-220.

McElhinney, L.M.; Fooks, A.R. and Radford, A.D. (2008): Diagnostic tools for the detection of rabies virus. European Journal of Companion Animal Practice. 18; 224-231.

Milius, J.; Jacevicius, E.; Tamosiunas, V.; Paulauskas, V. and Lukauskas, K. (2004): Rabies among Wild and Domestic Animals in Lithuania In 1993-2002: Epizootiology, Diagnosis And Vaccination. Bulletin of Veterinary Institute Pulawy 48; 195-200.

Olugasa, B.O.; Aiyedun, J.O. and Emikpe, B.O. (2011): Prevalence of Antibody against Rabies among confined, free roaming and stray dog in a transit city of Nigeria. Veterinaria Italiana. 47 (4) 453-460.

Ogunkoya, A.B.; Will, A.L. and Ezeokoli, C.D. (1984): Rabies in Oyo State in Nigeria: 19711982. International Journal of Zoonoses. 11: 84-94.

Okonko, I.O.; Adedeji, O.B.; Babalola, E.T.; Fajobi, E.; Fowotade, A. and Adewale, O.G. (2010): Why is their still rabies in the world? An Emerging Microbial and Global Health Threat. Global Veterinarian 4 (1); 34-50.

Office International Des Epizootics (2004): Manual of diagnostic test and vaccine for terrestrial animals (mammals, birds and bees) $4^{\text {th }}$ edition paris France. Part 2, Section 2.2, Pg 328-346.

Pastoret, P.P. and Kasppeler, A. (2004): European rabies control and its history. In: Historical Perspective of Rabies in Europe and the Mediterranean Basin. (Eds.), A.A. King, A.R. Fooks, M. Aubert and A.I.Wandeler. Paris, OIE: 325-335.

Ratzan, R.M. (2006): Rabies in Israel 2006: An Update and Review. Israel Journal of Emergency Medicine. 6 (3); 6-26.

Tepsumethanon, V.; Lumlertdacha, B.; Mitmoonpitak, C.; Sitprija, V.; Meslin, FX. and Wilde, $H$. (2004): Survival of naturally infected rabid dogs and cats. Clinical Infectious Diseases 39; 278-280.

Velasco-Villa, A.; Reeder, S.A.; Oreiori, L.A.; Yager, P.A.; Franka, R.; Blanto, J.D.; Zuckero, I.; Hunt, P.; Oertli, E.H.; Robinson, C.E. and Rupprecht, C.E. (2008): Enzootic rabies elimination from dog and reemergence in will terrestrial carnivores, United States. Emerging 
Infectious Disease. 14; 1849-1854.

Warrell, M.J. and Warrell, D.A. (2004): Rabies and other lyssavirus diseases. Lancet. 363; 959 969.

Wilkinson, L. (1988): Introduction, in Rabies. Edited by J.B. Campbell and K.M. Charlton. Boston: Kluwer Academic Publishers: 1-23.

Wilsmore, T.; Hamblin, C.; Taylor, N.; Taylor, W. and Watson, B. (2006): Qualitative Veterinary Risk Assessment of the Introduction of Rabies into the United Kingdom. A report prepared for the Department for Environment, Food and Rural Affairs (Defra).

World Health Organization (2010): Rabies. Zoonosis and Veterinary Public Health. World Health Organization, 2010.

Zienius, D.; Bagdonas, J. and Dranseika, A. (2003): Epidemiological situation of rabies in Lithuania from 1990 to 2000. Veterinary Microbiology. 93, 91-100. 